\title{
An evaluation of Unix in the psychology computer laboratory
}

\author{
DORIS AARONSON \\ New York University, New York, New York 10003
}

\begin{abstract}
Psychology users of the Unix operating system responded to questionnaire items concerning the use of Unix, the ease of learning and using Unix, and the value and quality of the system for their research needs. The data suggest that Unix is poor according to human-factors criteria, but good according to programming and systems criteria.
\end{abstract}

Unix $^{1}$ has been serving as an operating system on the PDP-11 and VAX machines in a few dozen psychology laboratories for the past few years. As this system is now easily available, and other psychologists are thinking of using it, it is appropriate to evaluate its effectiveness for our research needs. To obtain a data base on which to evaluate Unix, sets of three questionnaires were mailed to 25 computer-based psychology laboratories that are currently using Unix. Responses were received from 26 individuals in laboratories representing universities in New York, Illinois, Mississippi, Colorado, and California, along with several Bell psychology laboratories in three different New Jersey cities. The report on this questionnaire is divided into three parts: (1) who is using Unix and how it is being used in the psychology laboratory, (2) what the human being experiences when learning and interacting with Unix, and (3) the value of Unix software and the quality of its performance in our laboratories.

\section{WHO IS USING UNIX AND HOW IT IS BEING USED IN THE PSYCHOLOGY LABORATORY}

Figure 1 shows the types of people using Unix, the computers they are using, and the versions of Unix that they have implemented. The top panel shows that the largest group of users is the graduate student body. Although most of the questionnaires were sent to faculty, they were appropriately completed by the students and by salaried programmers (who are also generally students). These people do most of the laboratories' computer work on a daily basis. The distribution of types of users in this sample is probably typical of psychology Unix laboratories in general, with one exception: The category of secretaries is underestimated. Recipients of questionnaires were asked to give photocopies to secretaries. Although only one secretary completed a questionnaire, 15 other respondents answered

This work was supported in part by PHS Grant MH 16,496 to New York University. I thank Barry Cohen, Misha Pavel, Robert Sherak, and Nancy Weeks for comments on earlier drafts.
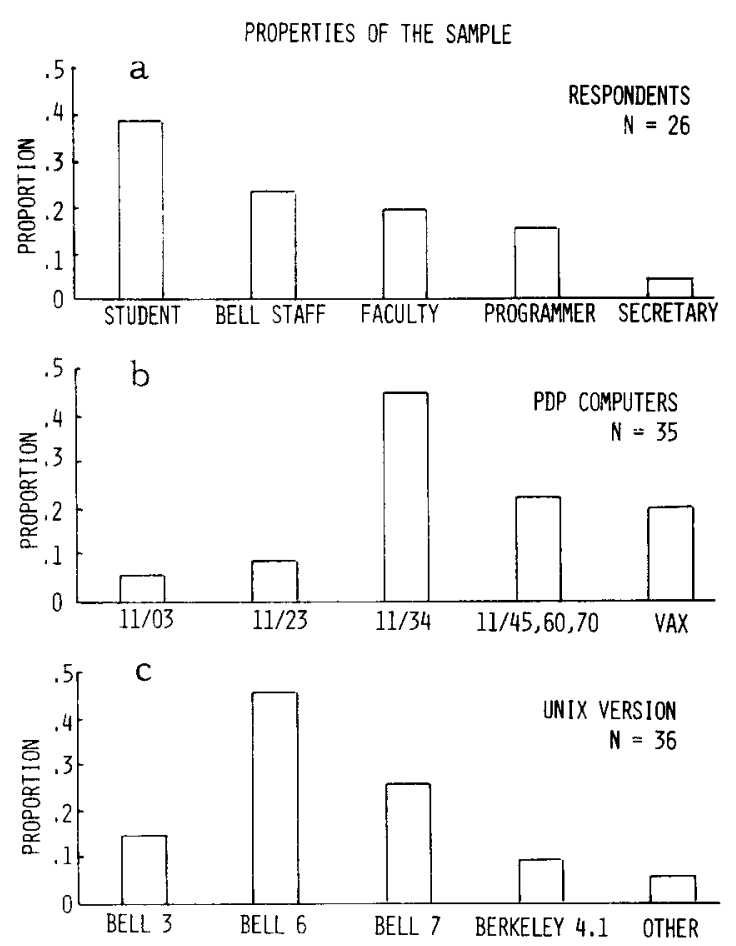

Figure 1. Properties of the present sample of respondents: (a) distribution of categories of Unix users, (b) distribution of DEC computers using Unix. (c) distribution of software version of Unix being used by the respondents.

the items on aspects of learning the system by a secretarial population. During the past year, my own secretary has used Unix more than anyone else in my laboratory, but she did not return the questionnaire after three requests. Perhaps she was afraid to give negative ratings of Unix or felt that those ratings would insult the faculty who selected Unix for her word processing duties. Laboratory heads should note this relative silence from a large Unix user population. The questionnaire data suggest that their computer needs, which differ from those of experienced programmers, are not adequately addressed.

For most of this report, responses will be grouped together for all categories of users. This grouping is 
acceptable because (1) respondents were asked to leave blank the items that they could not answer and (2) there were few, if any, differences in trends between user categories for items that were filled in.

The middle panel of Figure 1 shows that almost half the Unix users have the medium-sized PDP-11/34 in their laboratory. Note that (modified) Unix is operating on two of DEC's microcomputers, the 11/03 and 11/23. Also note that the distribution is weighted toward the larger machines, including a fair number of VAXs. This is because most versions of Unix require at least $128 \mathrm{~KB}$ of memory.

The bottom panel shows that almost half the users are working with the Bell Laboratories Version 6 of Unix. It appears that Bell Version 3 has generally not left Bell Laboratories. Although Bell Version 7 has been available for the past few years and is a marked improvement over Version 6, many users have not yet implemented it. In fact, Version 7 is now distributed commercially under the company name Xenix, including software support that Bell does not offer to non-Bell users. Berkeley Unix is superior to the others, especially on human engineering or "friendliness" criteria (Norman, in press), but it is so large that it will fit only on the 700 -series VAX machines. The category "other" includes "mini-Unix," designed for smaller machines. To summarize the data in Figure 1, the typical user is a psychology graduate student, with a PDP-11/34, using Bell Version 6 Unix.

Figure 2 shows the uses of Unix in psychology laboratories. These data are interesting in comparison with the situation 5 years ago, when most psychologists were using DEC systems on the PDP-8, 12, 9, and 15 . The top panel shows the proportion of respondents that use various programming languages. The predominant programming language is the $\mathrm{C}$ language, in which the Unix operating system itself is mostly written. This graph provides an underestimate of the use of $\mathrm{C}$, as it only includes proportions of users. The questionnaires asked for information on percentage of daily usage. The category could not be analyzed because some respondents merely checked the item instead of providing actual percentages. Of the 18 respondents reporting percentages, 15 use $\mathrm{C}$ over $50 \%$ of the time and 10 use $\mathrm{C}$ more than $90 \%$ of the time. Five years ago, no one in our groups used either $\mathrm{C}$ or PASCAL.

The data in the middle panel of Figure 2 are surprising, as the use of "laboratory" computers has changed drastically in 5 years. The predominant use is now word processing. Secretaries use the computers to type research reports, and graduate students use them to type theses. Five years ago, they used a device called a typewriter instead. The high proportion of data analysis also represents a change. Five years ago, most of us analyzed data on the university computer center IBMs, because the PDP-8s and 12 s were not large enough and assembler, FOCAL, and FORTRAN Il were nonoptimal languages for that type of work. Also, 5 years

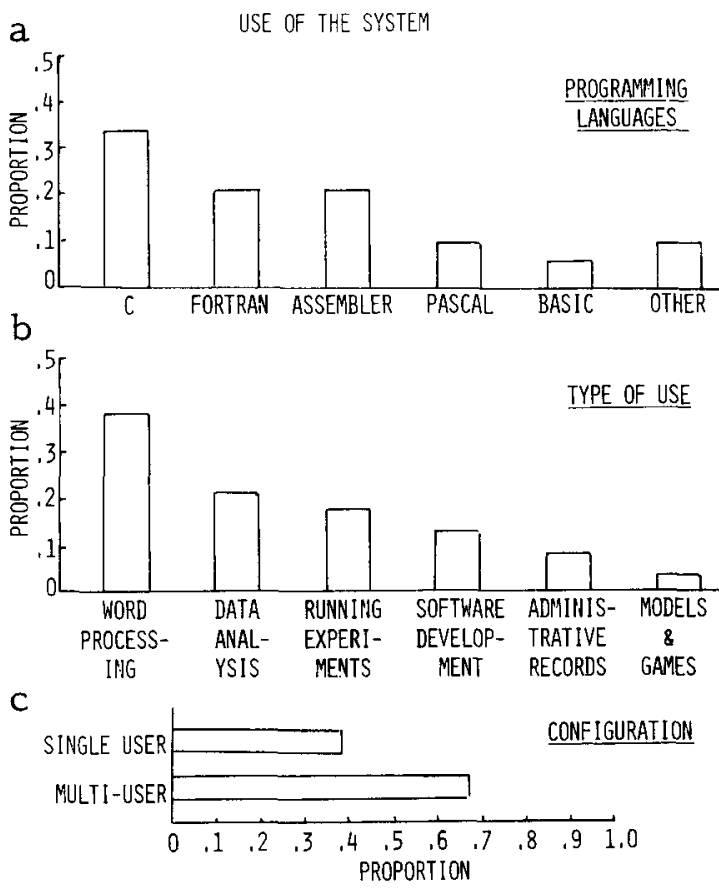

Figure 2. The ways Unix is used in psychology laboratories: (a) distribution of programming languages used by respondents, (b) distribution of research tasks being performed, (c) proportion of "stand-alone" vs. timesharing use of Unix machines.

ago, the bars labeled "administrative records" and "models and games" did not exist for small laboratory machines. A bothersome change is represented by the column "software development," because this type of work does not concern specific experiments. Some of that programming is related to keeping Unix operating smoothly. Also, the software development column includes general systems work needed to enable Unix to run real-time programs, a task for which it was not designed. In the "good old days," we spent most of our time and money being psychologists and DEC did the systems programming for us. Unix is so general, flexible, and "unfriendly" to inexperienced programmers that we are now spending large proportions of our time and money on systems programming rather than on activities more directly related to psychology.

The bottom part of Figure 2 shows the distribution of single-user computer use (generally for purposes of subject running), and timesharing or multiuser use (generally, the other categories indicated in the middle of Figure 2). The high proportion of multiuser time is consistent with the high amount of word processing being done.

\section{WHAT THE HUMAN BEING EXPERIENCES WHEN LEARNING AND INTERACTING WITH UNIX}

Figure 3 shows subjective ratings on a 10-point scale for ease of learning and using Unix, as well as the time needed to learn the system. The first panel shows 


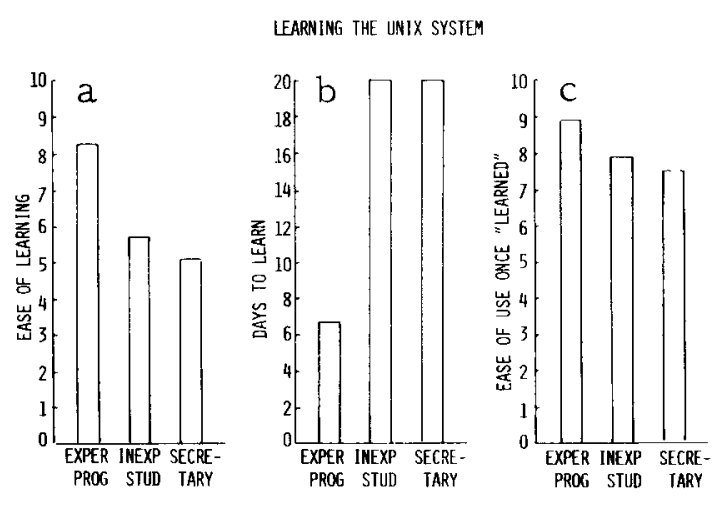

Figure 3. Ratings of Unix for: (a) ease of learning, (b) days to learn, and (c) ease of use by three types of users.

that Unix is rated about $50 \%$ easier to learn for experienced programmers than for inexperienced students and secretaries. The middle panel is consistent with the ratings, showing that inexperienced students and secretaries take about three times as long as experienced programmers to learn the system: about 20 days as opposed to 6.8 days. A quotation from a secretary rater illustrates the feelings of a newcomer to the computer world: "I feel more in control with a typewriter; nothing crashes and there is nothing to wait for." The third panel shows that once Unix has been learned, the ratings increase and the rating differences among groups markedly decrease.

Figure 4 shows some of the reasons why new programmers find Unix difficult to learn. The first panel shows that all users gave mediocre ratings to Unix documentation on all fronts: clarity, completeness, and organization. The last few questionnaire items were open-ended and asked that users list the specific problems with Unix that cause the most trouble. Consistent with the ratings, over half of the respondents listed poor documentation. The modifiers included "incomprehensible," "terse," "incomplete," "difficult to locate information," "unclear," and "without appropriate examples."

Panel 2 of Figure 4 shows that Unix scored somewhat higher on consistency of syntax, function operation, and command naming. On the open-ended items, only one-third of the respondents listed specific problems with consistency, but one-third is higher than one would like. Figure 5 shows some examples of Unix inconsistency taken from Norman's (in press) article in Datamation. Note the lack of consistency in relations between command names and functions. These "abbreviations," using from two to five letters, include the first two letters (as in "c compiler" and "editor"), the first two consonants (as in "copy" and "move"), the second syllable (as in "cat" for concatenate), much of the second word (as in "change password"), and no abbreviation at all (as in "date" or "echo"). The manual does not even tell us what the last abbreviation, "grep," stands for. Example B is just one instance of inconsistent syntax: The string-copy command in $\mathrm{C}$ and the file-copy command in Unix have the order of their arguments reversed. Example $\mathrm{C}$ shows that within only one part of Unix, the editor, a special symbol, period, can have four different meanings, depending on the mode and exact form of usage.
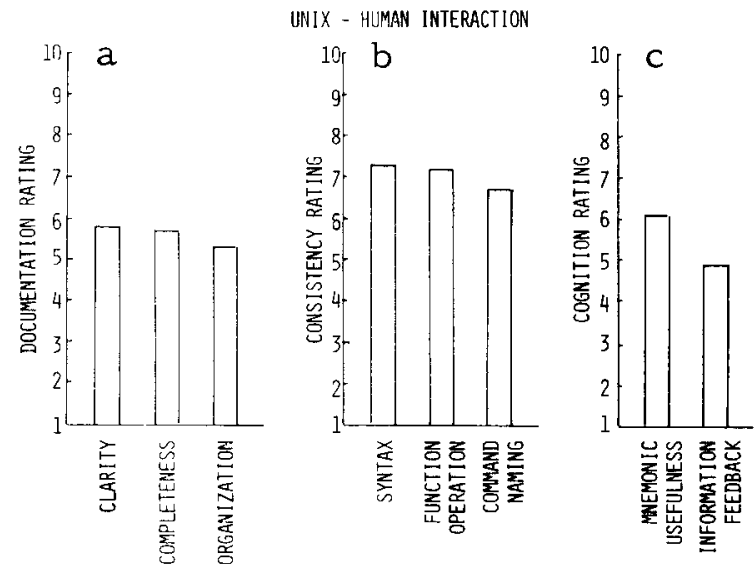

Figure 4. Ratings of the users' "information processing" experiences with Unix: (a) quality of documentation, (b) organization of command structures, (c) cognitive aspects of using the system.

A. LEXICAL RELATIONS BETWEEN FUNCTIONS \& NAMES

\begin{tabular}{ll} 
FUNCTION & UNIX COMMAND NAME \\
\cline { 2 - 2 } C COMPILER & CC \\
CHANGE WORKING DIRECTORY & CHDIR \\
CHANGE PASSWORD & PASSWD \\
CONCATENATE & CAT \\
COPY & CP \\
DATE & DATE \\
ECHO & ECHO \\
EDITOR & ED \\
MOVE & MV \\
SEARCH FILE FOR PATTERN & GREP
\end{tabular}

B. ARGUMENT ORDER IN SYNTAX

$\begin{array}{lll}\text { STRCPY (NEWSTRING, OLDSTRING) } \\ \text { CP } & \text { OLDFILE } & \text { NEWFILE }\end{array}$

C. MEANINGS OF THE SPECIAL SYMBOL "." (PERIOD)

THE CURRENT LINE

ANY CHARACTER

THE COMMAND TO EXIT APPEND MODE

END OF SENTENCE IN ENGLISH TEXT

Figure 5. Examples of three kinds of inconsistencies in the "user interface" of Unix: (A) abbreviations for commands, (B) argument order, (C) multiple meanings for symbols. (Based on Norman, in press.) 
Returning to Figure 4, mediocre ratings in the third panel show two more reasons why new programmers have trouble learning Unix. First, many of the command names are not good mnemonic choices for the functions they represent. On the open-ended items, about onethird of the respondents reported that a major problem was forgetting command names or details of command arguments. Second, Unix provides very little information feedback to the user. For example, there is no prompt symbol to let you know whether you are in editor, or if so, whether you are in editor's command or append mode. Many sections of Unix have little or nothing in the way of error messages to provide feedback on the user's mistakes. Finally, there is no warning to users when they are about to delete files. On the open-ended items, about one-third of the respondents complained about the lack of feedback, and, in addition, about one-third specifically and separately complained about unintentional file destruction due to lack of feedback warnings. I gasped when one user reported unintentional file destruction in another user's directories.

In summary, Figures 3, 4, and 5 suggest that Unix leaves much to be desired in terms of "human engineering" considerations, or just plain down-to-earth "friendliness" to the user. It is too bad that the Bell Laboratories programmers who designed the "user interface" of Unix did not seek the aid of some of the excellent Bell Laboratories psychologists who do research on human information processing. The questionnaire respondents almost unanimously supported Norman's (in press) opinions that the user interface of Unix is poor, according to human-factors criteria. But, in reality, we have little systematic empirical evidence (1) as to which criteria should predominate in the design of a complex, multidimensional system or (2) that any given criterion would markedly improve learning and use of a software system. These issues suggest an important and undeveloped area of research on human information processing.

\section{THE VALUE OF UNIX SOFTWARE AND THE QUALITY OF ITS PERFORMANCE IN PSYCHOLOGY LABORATORIES}

Figures 6 and 7 deal with Unix's ability to handle the psychologist's work. The users' responses in this respect are generally more favorable than those regarding the user interface. Unix is capable of handling many research needs, and users feel that it is superior to other operating systems in many ways.

In Figure 6, the major components of Unix are along the $\mathrm{x}$-axis. The $\mathrm{y}$-axis for the bottom panel indicates the frequency of users' responding to any given component. These frequency data are based on the assumption that when questionnaire slots were left blank for these components, the respondents did not use the

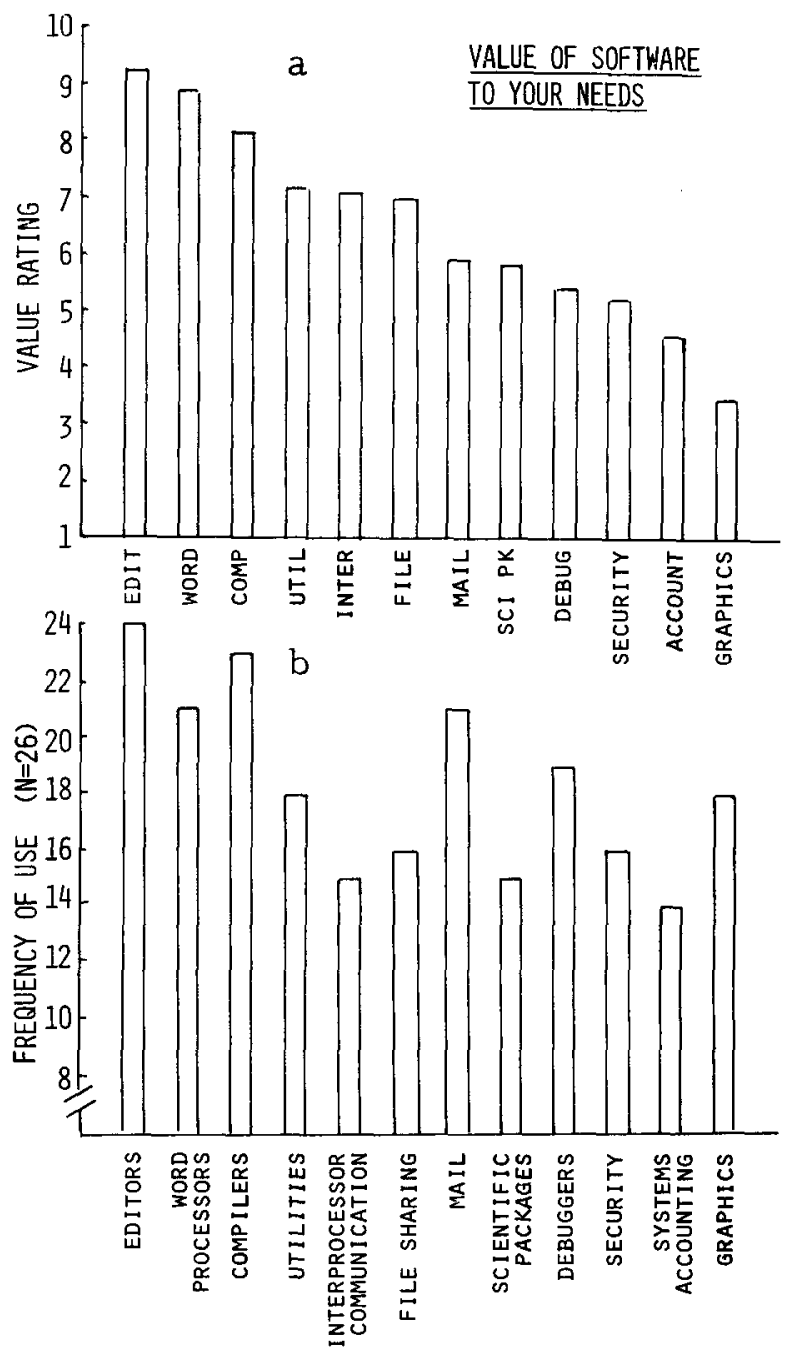

Figure 6. (a) Rated value and (b) frequency of use for software components in the Unix system.
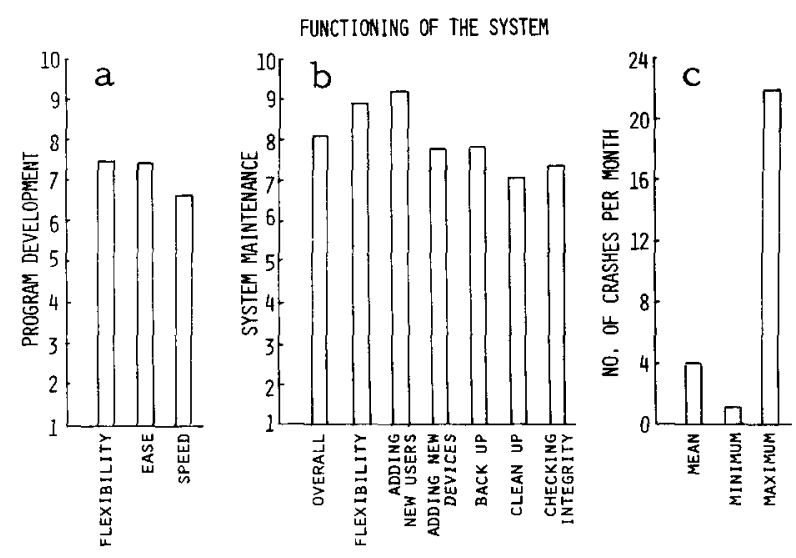

Figure 7. The quality of the Unix computer system during use: (a) program development, (b) system maintenance, and (c) number of crashes per month. 
components. The $y$-axis for the top panel is the mean value rating, given that the item was not left blank. Components were ordered from left to right in order of their mean rating value. Thus, consistent with the Figure 2 evidence that we are processing more words than subjects these days, the top panel of Figure 6 shows that the two most valuable components are the editors and word processors, such as Edit and Nroff. The next several components are necessary to run experiments and to analyze the resultant data. The last three items are mostly luxuries. Security is needed by realistically paranoid psychologists who fear others could destroy their files or change their class grading records. System accounting is needed to fulfill our compulsive needs for administrative records. And it is nice that graphics can make our data displays more aesthetic.

In comparing the top and bottom panels of Figure 6, four interesting points emerge. (1) The first three components are ranked highest whether the ranking is by frequency or by value. (2) We conduct a lot of "mail" communication, as indicated by the high frequency on the bottom panel, although this is not particularly valuable to our work, as indicated by the middling rating in the top panel. Perhaps the "mail" command will decrease the departmental photocopying costs for communication by "memos." (3) The debuggers are used a lot, although they are not rated high on the value scale. I point out this discrepancy because several users commented that the debugging tools were "terrible" on the open-ended questionnaire items. (4) Regardless of value, every component is used by at least half the respondents in our sample.

The first two panels in Figure 7 show some of the reasons why many users like Unix and would not trade it for any other operating system. The first panel shows that Unix is rated reasonably high on program development; the second shows that it is rated very high on some important systems features. The open-ended questionnaire items again support the rating data When asked to list the things they especially like about Unix, about $25 \%$ of the users listed each of the following four items: (1) the power of the "shell" programming features, (2) the ability to modify the operating system easily, (3) the $\mathrm{C}$ programming language, with its structured, powerful, and flexible commands, and (4) the hierarchical file structure. Surprisingly, in light of all the complaints about inconsistencies and file destruction with the editor, about $35 \%$ of the users listed editor as a Unix component that they especially like. Finally, $20 \%$ of the users especially like the I/O flexibility offered by the "piping" feature.

I was amazed that not a single respondent listed what
I consider to be the two most important reasons for liking Unix. First, it is virtually free. University and hospital research laboratories are charged a token fee of $\$ 230$, and Bell Laboratories researchers are obviously charged nothing. This is in comparison with thousands or tens of thousands of dollars that we would have to pay for an equivalent amount of DEC software for a psychology department that has several DEC computers. Second, Unix is an exceedingly powerful timesharing system--in essence, another budget factor. Up to 36 faculty members, students, and secretaries at a time can share the same computer, thereby reducing hardware costs.

The rightmost panel of Figure 7 indicates the number of crashes per month for Unix systems. The mean is 4.1. about 1 per week. But, as indicated, the data are highly variable, ranging from 1 to 22 . It appears that laboratories with lots of new users and laboratories with Bell Version 6 software have an inordinate number of systems crashes, almost one per day. For example, mounting an unformatted floppy disk with Version 6 causes a crash rather than an error message. Experienced users and Version 7 software have eliminated such problems.

In summary, the questionnaire data lead to two main conclusions. On the negative side, the human engineering properties of Unix are poor. The user interface is not "friendly" because (1) its commands, syntax, and functions are inconsistent. (2) it gives little in the way of help commands, error messages, and other feedback, and (3) the nonmnemonic command names are hard to learn and easy to forget. On the positive side, Unix is a high-quality computer tool in terms of program development and systems maintenance. It can handle our research needs, including running experiments, analyzing data, and writing reports, better than other existing computer systems. It appears that Bell Laboratories is making efforts to improve Unix, and many of the problems faced by the users have already been met in Version 7. Thus, we should see an expanded psychology user population for Unix in the years ahead.

\section{REFERENCE}

Norman, D. A. The truth about Unix: The user interface is horrid. Datamation, in press.

\section{NOTE}

1. Unix TM is a computer operating system developed by Dennis Ritchie and Ken Thompson of Bell Laboratories. It is trademarked by Bell Laboratories and is available under license from Western Electric. 